\title{
High Defibrillation Threshold: Brace For Impact
}

\author{
Karam Ayoub ${ }^{1}$ and Travis Richardson ${ }^{1}$ \\ ${ }^{1}$ Vanderbilt University Medical Center
}

November 18, 2021

\section{High Defibrillation Threshold: Brace For Impact}

Karam Ayoub, MD ${ }^{1}$; Travis Richardson, $\mathrm{MD}^{1} 1$. Division of Cardiac Electrophysiology, Vanderbilt University Medical Center, Nashville, TN, USA.

Disclosures : Dr. Richardson has received research funding from Medtronic Inc. and served as a consultant for Philips Inc. and Biosense-Webster Inc.

Conflict of interest : none

Funding : none

Key words : Defibrillator, Mortality, Defibrillation Threshold, Sudden Cardiac Death

Total word count: 1168

Corresponding author:

Travis D. Richardson, MD

Assistant Professor Cardiac Electrophysiology

Vanderbilt Heart and Vascular Institute

1215 21st Ave S. Nashville, TN

Medical Center East, South Tower, Suite 5209

travis.d.richardson@vanderbilt.edu

Sudden cardiac death (SCD) constitutes a major public health problem and accounts for approximately $50 \%$ of all cardiovascular deaths, including 230,000 to 350,000 deaths per year in the United States alone ${ }^{1}$. Affected by the death of his mentor, Michel Mirowski was dedicated to find a solution for such a medical problem. After years of work, he was able to build an implantable cardioverter-defibrillator (ICD), introduced in humans in 1980, and approved by the food and drug administration in $1985^{2}$. The ICD has transformed the treatment of patients at risk for sudden cardiac death due to ventricular tachyarrhythmias. Initially, implanting ICD leads required a thoracotomy, while the generator required an abdominal surgery given that it was large and bulky. Over the last 35 years, tremendous development in capacitors resulted in significant miniaturization of the ICD system and permitted subcutaneous pectoral implantation in most patients. In 2012, the FDA approved the first subcutaneous ICD, which opened the door to implant such life saving devices in specific populations without the option of, or at high risk for a transvenous approach. Not only are current ICDs smaller than early generations, they also have the functionality of low and high-energy shocks in multiple tachycardia zones along with antitachycardia pacing.

The transvenous ICD has been in clinical use for $>3$ decades, and robust data from high-quality randomized controlled trials support its use in various patient populations including survivors of cardiac arrest, patients 
with VT and structural heart disease, and patients with significant LV dysfunction ${ }^{3}$, leading to the increased use of ICD in these populations at risk for ventricular tachycardia. The role for defibrillation threshold (DFT) testing either intraoperatively or postoperatively has changed significantly over time. The definition of the DFT is a probabilistic value and is defined as the minimum energy required at which two shocks will both successfully terminate ventricular fibrillation. Such testing was routine at the time of all ICD implantations in the past due to uncertainty surrounding the device and a desire to better determine the probability of success in treating ventricular arrhythmias. Generally, devices are programmed with a safety margin of at least $10 \mathrm{~J}$, although some trial data indicates that a $5 \mathrm{~J}$ margin could provide equal efficacy ${ }^{4}$. However, when the safety margin is $<10 \mathrm{~J}$ or when the device fails to effectively terminate ventricular tachycardia, several interventions can be performed, including medical therapy, device reprogramming, or system revision/modification (table 1). However, there is limited data to assess the long-term outcomes of patient undergoing these modifications.

In this edition of the Journal of Cardiovascular Electrophysiology, Najmul et al. present a retrospective cohort study of 6353 patients undergoing ICD implantation and DFT testing; 191 of which had high DFT $($ mean $32.1+/-3.7 \mathrm{~J})$. High DFT was defined as $>25 \mathrm{~J}$ or within $10 \mathrm{~J}$ of maximal device output. During more than 2000 days of follow up, patients with high DFT had a higher mortality when compared to patients with acceptable DFT ( $48 \%$ vs $38 \%$, p =0.00046) with early separation in Kaplan Meier (KM) analysis. Patients with high DFT were more likely to be younger, taller, have non-ischemic cardiomyopathy, and lower ejection fraction.

In patients with high DFT, $63 \%$ (121/191) underwent system modification(SM) with approximately 10J decrease in DFT from baseline $(33.3+/-3.4 \mathrm{~J}$ to $24.8+/-5.9 \mathrm{~J}, \mathrm{P}=<0.001)$. A subcutaneous coil (Medtronic 6996SQ-58) was most commonly used (66\%). Interestingly, 17 / 121 patients required further device reprogramming and/or lead repositioning to attain a satisfactory DFT. Major complications of cardiac arrest, pulmonary edema, and shock were observed in $12 \%$ of patients who underwent SM vs $11 \%$ in patients with high DFT who did not undergo SM. Further, when compared to patients with high DFT who did not undergo SM to decrease DFT, patients who underwent SM had similar mortality rate during follow up (48\% vs 47 $\%, \mathrm{p}=0.91$ ). This held true in patients with both primary and secondary prevention indications. Notably, sudden/arrhythmic death could not be adjudicated in this dataset.

DFT at the time of initial ICD placement was standard of care for many years. With the advent of biphasic waveforms and high output shocks, which result in more reliable defibrillation, the value of routine DFT testing was revisited ${ }^{5}$. This question was addressed in the SAFE-ICD study where the primary end point (composite of severe complications at ICD implant and sudden death or resuscitation at 2 years) was similar in patients who underwent DFT testing and those who did not ${ }^{6}$. Interestingly, in the SAFE-ICD study, $<7 \%$ of patients had high DFT testing, which required an intervention, similar to what was observed in the study by Najmul et al. In light of these findings there has been a significant reduction in the routine performance of DFT testing at the time of ICD implantation ${ }^{7}$. The findings of Najmul et al. further support avoidance of routing DFT testing, especially if corrective measures may not affect outcomes in general. This raises the question of why, despite effectively reducing the DFT, SM did not affect outcomes. It seems clear that patients with high DFT represent a high-risk population independent of their risk of arrhythmic death. One interesting observation was that the KM curves separate early during follow up between patients with high DFT compared to acceptable DFT, raising the question of whether intra-procedural or post-procedural complications could have balanced out any reduction in sudden death risk afforded by SM. Indeed, patients with high DFT had a $12 \%$ complication rate, likely related to prolonged procedure times, lead revisions, and repeated inductions (median 3, with range between 1 to 12). Importantly, these data cannot be generalized to patients in which ICD therapy has failed to successfully treat a clinically observed ventricular arrhythmia, as that population was not included and is likely to have a much higher risk of recurrent sudden death.

The study by Najmul et al. further supports the avoidance of routine DFT testing with current generation ICDs. These data raise doubt that system modification affects outcomes, potentially because any decreased risk of arrhythmic death may be balanced by increased procedural risk within what is already a high-risk population. However, in a certain subset of patients with high risk of both sudden death and high DFT, and 
certainly in those with unsuccessful treatment of clinical events, system modification to improve defibrillation efficacy should still be considered.

Table 1 : Methods to decrease DFT, Least to Most Invasive

\begin{tabular}{|c|c|c|}
\hline Intervention & Description & Reduction in DFT \\
\hline Reversal of Shock Polarity ${ }^{8}$ & Can to Coil vs. Coil to Can & $15-30 \%$ \\
\hline Changing Shock Waveform ${ }^{9}$ & $\begin{array}{l}\text { Available with certain } \\
\text { companies. }\end{array}$ & $3-5 \mathrm{~J}$ \\
\hline Medical therapy ${ }^{10,11}$ & $\begin{array}{l}\text { Sotalol and Dofetilide decrease } \\
\text { DFT. }\end{array}$ & $\begin{array}{l}\sim 4-5 \mathrm{~J} \text { with Sotalol } \sim 20 \% \text { with } \\
\text { Dofetilide }\end{array}$ \\
\hline SVC coill $^{12,13}$ & $\begin{array}{l}\text { Must be used at initial } \\
\text { implant or requires lead } \\
\text { replacement. }\end{array}$ & $2-3 \mathrm{~J}$ \\
\hline RV ICD lead revision ${ }^{13}$ & $\begin{array}{l}\text { Apical RV lead location may } \\
\text { reduce DFT, RVOT with septal } \\
\text { coil alternative }\end{array}$ & NA \\
\hline Addition of other coils $12,14,15$ & $\begin{array}{l}\text { Azygous, coronary sinus, } \\
\text { subcutaneous array/coil }\end{array}$ & Insufficient data \\
\hline
\end{tabular}

1. Deo R, Albert CM. Epidemiology and genetics of sudden cardiac death. Circulation . 2012;125(4):620-637. doi:10.1161/CIRCULATIONAHA.111.023838

2. Mirowski M, Reid PR, Mower MM, et al. Termination of malignant ventricular arrhythmias with an implanted automatic defibrillator in human beings. $N$ Engl $J$ Med . 1980;303(6):322-324. doi:10.1056/NEJM198008073030607

3. Woods B, Hawkins N, Mealing S, et al. Individual patient data network meta-analysis of mortality effects of implantable cardiac devices. Heart Br Card Soc . 2015;101(22):1800-1806. doi:10.1136/heartjnl2015-307634

4. Gold MR, Higgins S, Klein R, et al. Efficacy and temporal stability of reduced safety margins for ventricular defibrillation: primary results from the Low Energy Safety Study (LESS). Circulation . 2002;105(17):2043-2048. doi:10.1161/01.cir.0000015508.59749.f5

5. Neuzner J, Pitschner HF, Huth C, Schlepper M. Effect of biphasic waveform pulse on endocardial defibrillation efficacy in humans.Pacing Clin Electrophysiol PACE . 1994;17(2):207-212. doi:10.1111/j.15408159.1994.tb01373.x

6. Brignole M, Occhetta E, Bongiorni MG, et al. Clinical evaluation of defibrillation testing in an unselected population of 2,120 consecutive patients undergoing first implantable cardioverter-defibrillator implant. $J$ Am Coll Cardiol . 2012;60(11):981-987. doi:10.1016/j.jacc.2012.05.014

7. Borne RT, Randolph T, Wang Y, et al. Analysis of Temporal Trends and Variation in the Use of Defibrillation Testing in Contemporary Practice.JAMA Netw Open . 2019;2(10):e1913553. doi:10.1001/jamanetworkopen.2019.13553

8. Olsovsky MR, Shorofsky SR, Gold MR. Effect of shock polarity on biphasic defibrillation thresholds using an active pectoral lead system.J Cardiovasc Electrophysiol . 1998;9(4):350-354. doi:10.1111/j.15408167.1998.tb00923.x

9. Denman RA, Umesan C, Martin PT, et al. Benefit of millisecond waveform durations for patients with high defibrillation thresholds. Heart Rhythm . 2006;3(5):536-541. doi:10.1016/j.hrthm.2006.01.027 
10. Dopp AL, Miller JM, Tisdale JE. Effect of drugs on defibrillation capacity. Drugs . 2008;68(5):607-630. doi:10.2165/00003495-200868050-00004

11. Bollmann A, Husser D, Cannom DS. Antiarrhythmic drugs in patients with implantable cardioverterdefibrillators. Am J Cardiovasc Drugs Drugs Devices Interv . 2005;5(6):371-378. doi:10.2165/00129784200505060-00004

12. Kroll MW, Schwab JO. Achieving low defibrillation thresholds at implant: pharmacological influences, RV coil polarity and position, SVC coil usage and positioning, pulse width settings, and the azygous vein.Fundam Clin Pharmacol . 2010;24(5):561-573. doi:10.1111/j.1472-8206.2010.00848.x

13. Gold MR, Olsovsky MR, Pelini MA, Peters RW, Shorofsky SR. Comparison of single- and dual-coil active pectoral defibrillation lead systems.J Am Coll Cardiol . 1998;31(6):1391-1394. doi:10.1016/s07351097(98)00103-x

14. Faheem O, Padala A, Kluger J, Zweibel S, Clyne CA. Coronary sinus shocking lead as salvage in patients with advanced CHF and high defibrillation thresholds. Pacing Clin Electrophysiol PACE . 2010;33(8):967972. doi:10.1111/j.1540-8159.2010.02726.x

15. Kühlkamp V, Dörnberger V, Mewis C, Seipel L. Comparison of the efficacy of a subcutaneous array electrode with a subcutaneous patch electrode, a prospective randomized study. Int J Cardiol . 2001;78(3):247256. doi:10.1016/s0167-5273(01)00381-3 